\title{
Forrajeo de abejas sobre cultivos de soja, una aproximación mediante análisis de cargas corbiculares en Apis mellifera
}

\author{
Forage of bees on soy crops, an approach by analysis \\ of corbicular loads in Apis mellifera
}

\author{
D.C. Blettler ${ }^{1 *}$, D.M. Chémez ${ }^{1}$, P.L. Soñez $z^{1}$ M.A. Galloํ, G.A. Fagúndez $z^{1}$
}

\section{RESUMEN}

Las interacciones entre las especies vegetales y sus polinizadores resultan trascendentales en el ámbito de la biología y adquieren también singular importancia en su dimensión agroproductiva, tanto para cultivos que demanden servicios de polinización como para los que requieran aplicaciones fitosanitarias durante su floración, especialmente si de estas flores las abejas extraen polen, dado que este producto (fuente de proteína de las abejas) registra generalmente mayor contaminación química que el néctar. En consecuencia, es útil conocer y eventualmente predecir el comportamiento forrajero de las abejas sobre el cultivo de soja, debido a su mayoritaria participación en la matriz agroproductiva argentina. Se propone una aproximación metodológica novedosa que consiste en capturar abejas durante el ingreso a las colmenas con redes entomológicas y posterior análisis palinológico de sus cargas corbiculares (se ponderó el aporte porcentual de soja, respecto de otros recursos, con el número de abejas atrapado en cada repetición). Las capturas se realizaron secuencialmente en colmenas lindantes con un lote de soja durante su periodo de floración, cada 2 días y en 6 horarios (9:00, 10:30, 12:00, 13:30, 15:00 y 16:30 h), con 3 repeticiones por horario. Los resultados indican que las abejas prefieren forrajear polen de soja en horarios comprendidos entre las 12:00 am y las 15:00 pm con un máximo de actividad recolectora sobre las $13: 30 \mathrm{pm}(\mathrm{p}<0,001)$. También se destaca un progresivo aumento en la preferencia por el polen de soja a medida que avanza la floración del cultivo, registrando valores máximos después de su plena floración $(\mathrm{p}<0,001)$. Los resultados están multifactorialmente condicionados por la oferta floral del cultivo, la simultaneidad de otras floraciones alternativas y por condiciones meteorológicas.

Palabras clave: soja, abejas melíferas, polen, comportamiento.

\begin{abstract}
The interactions between plant species and their pollinators are transcendent in the field of biology and also acquire singular importance in their agro-productive dimension; both for crops that demand pollination services and for those that require phytosanitary applications during their flowering, especially if from these flowers bees extract pollen, given that this product (source of protein from bees) generally registers greater chemical contamination than the nectar. Consequently, it is useful to know and eventually predict the foraging behavior of bees on soybean cultivation, given their majority participation in the Argentine agro-productive matrix. A novel methodological approach is proposed that consists of capturing bees during entry to the hives with entomological nets and subsequent palynological analysis of their corbicular loads (the percentage contribution of soybeans was weighted, concerning to other resources, with the number of bees trapped in each repetition). The captures were made sequentially in hives bordering a batch of soybeans during its flowering period, every 2 days and at 6 times (9:00, 10:30, 12:00, 13:30, 15:00 and 16:30 h), performing 3 repetitions per schedule. The results indicate that the bees prefer to forage soybean pollen at times between 12:00 AM and 15:00 PM with a maximum of collecting activity around 13:30 PM $(p<0.001)$. It also highlights a progressive increase in the preference for soybean pollen as the crop progresses, registering maximum values after its full bloom $(p<0.001)$. The results are multifactorially conditioned by the floral offer of the crop, the simultaneity of other alternative blooms and by meteorological conditions.
\end{abstract}

Keywords: soybean, honeybees, pollen, behavior.

\footnotetext{
1 Laboratorio de Actuopalinología. CICYTTP (CONICET-PROV. E.R.-UADER). Diamante, Entre Ríos, Argentina.

* Autor por correspondencia: dcblettler@hotmail.com
}

Fecha de Recepción: 7 de Agosto, 2020.

Fecha de Aceptación: 30 de Septiembre, 2020. 


\section{Introducción}

La relación entre las diferentes especies vegetales y sus polinizadores es una interacción trascendente en el ámbito de la biología (Hoiss et al., 2015). Asimismo, para el particular ámbito de incumbencias de la agronomía redunda en aumentos de rendimientos sobre muchas especies vegetales de interés agrocomercial (Rader et al., 2013), además de beneficiar a la producción apícola con recursos florales estratégicos.

El conocimiento acabado de las interacciones (planta - polinizador) permite ajustar detalles durante la introducción de colmenas en inmediaciones de los cultivos agrícolas, forestales o forrajeros (sea con finalidad de polinización o cuando se utiliza a los cultivos como proveedores de recursos alimentarios para las colmenas) y posibilita también la reducción de los riegos para las abejas y otros polinizadores durante las pulverizaciones fitosanitarias (Blettler et al., 2016).

Del amplio conjunto de insectos polinizadores, las abejas melíferas (Apis mellifera L.) son los polinizadores más valiosos desde el punto de vista económico para la agricultura (Klein et al., 2007) y particularmente en el cultivo de soja son también los polinizadores potenciales más abundantes (Fagúndez et al., 2016) y responsables fundamentales de significativos aumentos del rendimiento del cultivo (Blettler et al., 2018).

En los ambientes agriculturizados, el polen extraído de las flores por las abejas para garantizar la ingesta proteica de las crías ha sido definido en numerosos trabajos científicos como la vía de mayor ingreso de contaminantes químicos a las colmenas (Mullin et al., 2010). Las abejas colectan polen de una diversidad de plantas a diferentes horas del día dependiendo, entre otros factores, de la disponibilidad en cada flor y de los patrones diarios de temperatura que pueden afectar la actividad de vuelo de las abejas y la apertura de las anteras (Shawer, 1987). Para el caso particular del cultivo de soja, la recolección de polen por las abejas melíferas está relacionada, además de los anteriores factores, con la capacidad de la variedad para producirlo. En algunas regiones pueden ser colectadas pequeñas cantidades, mientras que en otras, hasta un $85 \%$ del total de polen recolectado por la misma colonia puede provenir de la soja (Free, 1993).

Numerosos trabajos científicos han abordado estudios tendientes a mejorar la comprensión de esta simbiosis entre plantas e insectos (Abrol, 2007) aportando, como en este trabajo, al esclarecimiento de los horarios predilectos de forrajeo de las abejas sobre diferentes cultivos (Torretta et al., 2010). Sin embargo, muy pocos estudiaron el comportamiento recolector de Apis mellifera específicamente sobre el cultivo de soja (Fagúndez et al., 2016), a pesar de su mayoritaria prevalencia en el ambiente agropecuario argentino (Viglizzo et al., 2010).

En las investigaciones previas que han abordado como objeto de estudio las interacciones entre insectos polinizadores y cultivos se han usado diferentes herramientas para analizar el comportamiento forrajero de las abejas.

- Determinando el origen botánico de las cargas corbiculares retenidas por trampas de polen antepuestas al ingreso de las abejas a sus respectivas colmenas (Basilio, 2000).

- Mediante la cuantificación de insectos por observaciones directas sobre las flores de los cultivos, en determinados momentos del día y por espacios más o menos prolongados de tiempo (Torretta et al., 2010).

- Mediante capturas usando redes entomológicas sobre la canopia del cultivo (Blettler et al., 2016; Rodríguez Parilli, 2014).

Cualquiera sea el método empleado, presenta limitantes que podrían sub o sobrevaluar los resultados forzando errores en su interpretación.

El uso de trampas de polen puede influir en el comportamiento forrajero de la colonia (Dreller et al., 1999). Además de este inconveniente, la bibliografía cita tamaños de cargas corbiculares muy variables (Harano y Sasaki, 2015) que dependen de la especie vegetal visitada y de las condiciones meteorológicas en que ocurrió la colecta de ese polen (Vaissière et al., 1994). Este hecho provoca sobre o subestimación del ingreso de polen, dado que las trampas retienen más fácilmente las cargas de mayor tamaño y son permeables a las más pequeñas.

Si el muestreo de forrajeo sobre un determinado cultivo se realiza por observación directa o con cámara filmadora, es posible discernir, aunque con dificultad, si la visita tiene como finalidad la colecta de néctar y/o polen observando el comportamiento recolector de las abejas (Gurini y Basilio, 1995). Sin embargo, el comportamiento que permitiría inducir la colecta de uno u otro recurso, es decir, 
néctar (introducción de la cabeza o la lengua en la flor) y polen (movimientos de barrido, cepillado y recolección, llevando polen en sus corbículas) debiera ser validado para cada especie vegetal sobre la que se observe. No puede generalizarse tan fácilmente.

Los datos proporcionados mediante la utilización de redes entomológicas para las capturas de insectos polinizadores presentan las limitantes de la técnica anterior y se le suma la dificultad de distinguir a los visitantes florales del total de insectos atrapados en las redes.

Para enmendar, en parte, estas falencias metodológicas y mejorar la comprensión respecto de las preferencias horarias de Apis mellifera en la colecta de polen de soja, en el estudio se utilizó un método novedoso que prevé reducir los errores planteados.

\section{Materiales y métodos}

La metodología empleada en este trabajo es inédita, pensada y desarrollada solo a los fines de obtener información fidedigna relativa a los objetivos planteados. En la investigación se estudió el polen corbicular de las abejas que ingresaban a las colmenas (prescindiendo del uso de trampas de polen) en diferentes horarios y se cuantificó la relación porcentual del polen correspondiente al cultivo aledaño (soja), respecto del total de polen ingresado en cada momento a las colmenas.

\section{Área de estudio}

El ensayo se realizó sobre un apiario (18 colmenas) contiguo a un lote de soja (Glycine max) de 20 hectáreas situado en la localidad de Oro Verde ( $31^{\circ} 50^{\prime} \mathrm{S}$ y $60^{\circ} 30^{\prime} \mathrm{O}$, a 95 metros de altitud), departamento Paraná, Entre Ríos.

La experiencia, en su fase de campo, fue conducida en la campaña agrícola 2014-2015. El cultivo antecesor en el lote experimental fue avena (Avena sativa) y la soja se sembró en noviembre de 2014, a $52 \mathrm{~cm}$ de distancia entre surcos y con 27 semillas por metro lineal de surco. Se utilizó el cultivar Nidera A 5009 RG.

Durante el ciclo del cultivo solo se realizaron aplicaciones sanitarias (tratamiento contra lepidópteros) cuando fueron superados los Umbrales de Daño Económico (UDE) en fecha 30 de enero. Este hecho anticipó ligeramente el fin de la experiencia, ya que los insecticidas aplicados podrían interferir en el normal desarrollo de las actividades de pecoreo de las abejas alterando el comportamiento y distorsionando los resultados.

Durante el desarrollo de la experiencia se relevó la flora alternativa en el área de influencia del apiario, la cual estuvo conformada mayoritariamente por Medicago sativa L. en lotes implantados; y en banquinas, alambrados y/o accesos, por especies herbáceas adventicias como Melilotus albus Desr., Cirsium vulgare (Savi) Tenore, especies de Carduus, Centaurea melitensis L., Cichorium intybus L., Ammi visnaga (L.) Lam., A. majus L., así como hierbas y arbustos nativos (especies de Bidens, Solidago chilensis Meyen var. chilensis, especies de Adesmia, Aloysia gratissima (Gill. et Hook.) Troncoso var. gratissima, Baccharis punctulata DC., B. dracunculifolia DC. y Asparagus officinalis L.), además de árboles exóticos naturalizados de Ligustrum lucidum Aiton.

\section{Mecánica de captura de abejas}

Sobre tres colmenas al azar (de un total de 18 que conformaron el apiario) se procedió a cerrar piqueras transitoriamente mediante el uso de un lienzo, previo ahumado de ellas. Transcurridos 60 segundos de esta acción se procedió a capturar con una red (por 5 segundos) las abejas que intentaban ingresar a su colmena pero no podían por encontrar cerrada la piquera. Estas capturas se realizaron cada dos días totalizando nueve fechas de muestreo durante la floración del cultivo (solo una captura fue alterada por la ocurrencia de lluvias el 28 de enero). Las capturas se hicieron con red entomológica de $38 \mathrm{~cm}$ de diámetro, en seis horarios diarios (9:00; 10:30; 12:00; 13:30; 15:00 y 16:30 h). En estas mediciones se tomó en consideración el horario oficial argentino GTM -3 (Greenwich Mean Time) que está una hora corrido con respecto al huso horario en que se encuentra localizada la región de muestreo, GTM-4 (Figura 1).

Finalmente, las abejas capturadas fueron dispuestas en bolsas plásticas, rotuladas y conservadas en freezer hasta su posterior recuento y análisis en laboratorio de sus cargas de polen.

\section{Análisis de laboratorio}

Se puso a punto una técnica para concentrar la mayor cantidad posible de material: cada una 


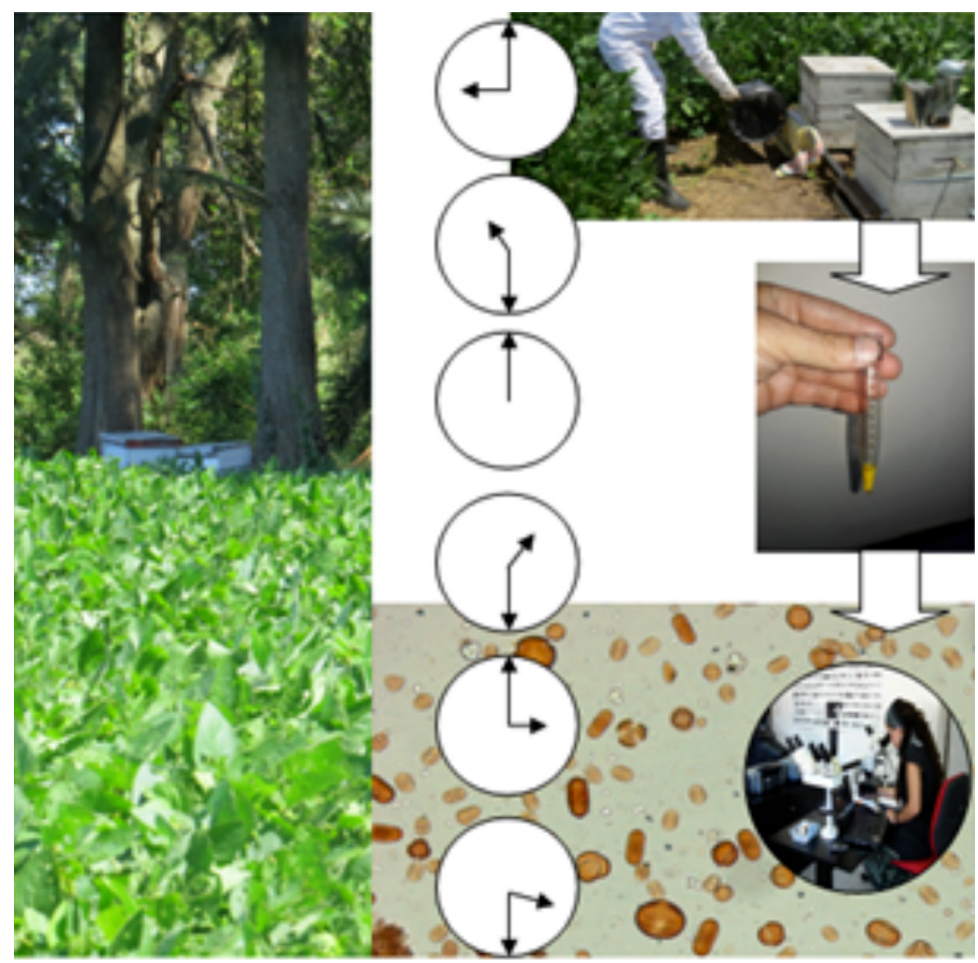

Figura 1. Resumen gráfico que refleja la secuencia cronológica (relojes en el centro de la imagen) de las capturas de abejas realizadas en este trabajo y muestra cómo las abejas fueron atrapadas con una red durante la entrada en los cajones (margen superior derecho).

de las repeticiones recibió el siguiente tratamiento para extraer (aislar) el polen que se procesará:

La bolsa y las abejas fueron lavadas con agua a $80{ }^{\circ} \mathrm{C}$ y detergente no iónico en un vaso de precipitado. Este caldo se dejó reposar por 24 horas. Posteriormente el total del líquido fue trasvasado a tubos de centrifugado (para concentrar el polen) a $1000 \mathrm{~g}$ durante 10 minutos. Una vez finalizado, se pasó el residuo a un tubo de $10 \mathrm{ml}$ para su ulterior procesamiento mediante acetólisis con posteriores centrifugaciones y lavados $(1000 \mathrm{~g}$ durante 2.5 minutos). Una alícuota de la muestra fue montada sobre un portaobjeto en glicerina fenolada y sellada con parafina.

Los granos de polen fueron observados bajo microscopio óptico Leica DME, ubicado en el Laboratorio de Actuopalinología del CICYTTP. Se realizó el recuento de 1200 granos, separando los de soja de los restantes. El conteo se hizo sobre transectas lineales (líneas equidistantes paralelas determinadas por los bordes superior e inferior del cubreobjetos y distribuidas desde el borde izquierdo al derecho de éste). El conteo se expresó en porcentaje de granos de soja versus el resto de los contabilizados.

\section{Interpretación de resultados}

La noción de promedio ponderado se utiliza para nombrar un método de cálculo que se aplica cuando, dentro de una serie de datos, alguno de ellos tiene una importancia mayor. Hay, por lo tanto, algún dato con mayor "peso" que el resto. El promedio ponderado consiste en establecer dicho "peso", también conocido como ponderación. En este trabajo se asume que la proporción resultante de granos de polen de soja, respecto del total de granos relevados, ponderado por la cantidad de abejas capturadas en cada muestra, es orientativa de la predilección de las abejas por las flores de soja, valor al que hemos denominado PREFERENCIA. Para esto primeramente se multiplicó cada dato (\% de polen de soja de cada captura/repetición) por su ponderación (cantidad de abejas capturadas en cada repetición). Luego se sumaron los valores asî obtenidos de cada horario y finalmente se dividió 
esta suma por el número de repeticiones que conforma la muestra de cada horario evaluado (tres).

Este valor está condicionado por la disponibilidad de recursos poliníferos en cada horario evaluado y por las condiciones climáticas imperantes en cada momento, y constituye un reflejo del comportamiento recolector de polen de las abejas melíferas frente al estímulo de un campo de soja en floración.

\section{Análisis estadístico de los resultados}

Para determinar diferencias significativas entre las medias aritméticas de los distintos tratamientos se ejecutaron análisis ANOVA de un factor, considerando diferencias significativas cuando $\mathrm{p}<0,05$. Pruebas post hoc de Fisher fueron utilizadas para explorar entre cuáles de los tratamientos hubo diferencias significativas. Posteriormente, se trazaron gráficos de barra y curvas a fin de mostrar densidades medias entre los distintos tratamientos de acuerdo a los objetivos. Se empleó el software INFOSTAT (Di Rienzo et al., 2011) para el análisis estadístico de los datos y para la construcción de los gráficos.

\section{Resultados y discusión}

En la Tabla 1 se observan los valores registrados de porcentaje promedio de polen de soja con respecto al número total de granos contabilizados, el número de abejas / captura y la preferencia por el polen de soja en tratamientos que incluyen diferentes horas de entrada de abejas a las colmenas y diferentes fechas de captura en relación con el ciclo fenológico de la floración de la soja.

Se evidencia que la preferencia por el polen de soja muestra valores muy diferentes durante el período horario evaluado de cada uno de los días de recolección de la muestra (Figura 2A). Esto está relacionado con la fluctuación de la actividad forrajera de las abejas y posiblemente también con la oferta de polen del cultivo de soja.

Otro resultado interesante que vemos en la misma figura (Figura 2A) es el aumento creciente en la preferencia de recolección de polen de soja desde el inicio de la prueba, cuando el cultivo tuvo muy poca floración, hasta el final de la prueba cuando ya había excedido incluso el número máximo de flores del cultivo que se produjo el 24 de enero.

Tabla 1. Diferencias y desviaciones estándar de las variables relevadas.

\begin{tabular}{clll}
\hline Tratamiento & \multicolumn{1}{c}{$\begin{array}{c}\text { \% de polen } \\
\text { de soja }\end{array}$} & \multicolumn{1}{c}{$\begin{array}{c}\text { Número abejas/ } \\
\text { captura }\end{array}$} & \multicolumn{1}{c}{ Preferencia } \\
\hline \multicolumn{5}{c}{ Horario/captura } \\
\hline $9: 00$ & $0,36(0,69) \mathrm{A}$ & $28,30(19,46) \mathrm{A}$ & $0,12(0,25) \mathrm{A}$ \\
$10: 30$ & $11,36(14,66) \mathrm{AB}$ & $36,88(17,60) \mathrm{AB}$ & $3,40(5,17) \mathrm{A}$ \\
$12: 00$ & $33,25(33,44) \mathrm{C}$ & $49,63(27,96) \mathrm{C}$ & $17,19(14,5) \mathrm{BC}$ \\
$13: 30$ & $47,11(31,83) \mathrm{C}$ & $42,41(25,64) \mathrm{BC}$ & $21,98(18,53) \mathrm{C}$ \\
$15: 00$ & $35,71(30,60) \mathrm{C}$ & $53,26(23,27) \mathrm{C}$ & $18,37(15,94)$ \\
$16: 30$ & $15,44(29,65) \mathrm{B}$ & $31,71(18,94) \mathrm{AB}$ & $\mathrm{C} 6,09(11,65) \mathrm{AB}$ \\
\hline \multicolumn{5}{c}{$\mathrm{Fecha} / \mathrm{Captura}$} \\
\hline $12 / 01$ & $5,25(10,02) \mathrm{A}$ & $34,72(19,26) \mathrm{AB}$ & $1,96(2,2) \mathrm{A}$ \\
$14 / 01$ & $24,97(31,57) \mathrm{BC}$ & $30,06(16,72) \mathrm{A}$ & $7,21(9,38) \mathrm{AB}$ \\
$16 / 01$ & $22,15(24,55) \mathrm{ABC}$ & $43,61(20,62) \mathrm{ABC}$ & $10,64(11,48) \mathrm{AB}$ \\
$18 / 01$ & $14,04(18,99) \mathrm{AB}$ & $44,72(32,11) \mathrm{ABC}$ & $7,24(8,35) \mathrm{AB}$ \\
$20 / 01$ & $5,71(6,14) \mathrm{A}$ & $29,61(26,92) \mathrm{A}$ & $2,05(2,39) \mathrm{A}$ \\
$22 / 01$ & $26,92(34,29) \mathrm{BC}$ & $39,28(20,12) \mathrm{ABC}$ & $11,86(14,49) \mathrm{AB}$ \\
$24 / 01$ & $36,60(33,75) \mathrm{CD}$ & $50,89(20,91) \mathrm{C}$ & $17,02(14,67) \mathrm{AB}$ \\
$26 / 01$ & $48,43(31,89) \mathrm{D}$ & $47,47(24,45) \mathrm{BC}$ & $21,28(17,10) \mathrm{B}$ \\
$29 / 01$ & $36,97(42,32) \mathrm{CD}$ & $47,27(27,05) \mathrm{BC}$ & $21,48(27,33) \mathrm{B}$ \\
\hline
\end{tabular}

Porcentaje de polen de soja con respecto al total, número de abejas / captura y preferencia por el polen de soja en diferentes momentos de entrada de abejas a las colmenas y diferentes fechas de captura en relación con la fenología de la floración de la soja. Los valores entre paréntesis que acompañan al valor medio de cada variable indican la desviación estándar y las diferentes letras expresan diferencias significativas utilizando la prueba de Fisher $(\alpha=0,05)$. 
Según reportan trabajos anteriores (que evaluaron presencia de polinizadores en canopia de soja), se esperaría que la preferencia por la recolección de polen de soja acompañe fielmente el suministro de flores del cultivo que será máximo en el pico de floración (Blettler et al., 2016; Fagúndez et al., 2016). Sin embargo, aunque la tendencia, se observan disminuciones de este indicador el 20 de enero y un pico de recolección el 29 de enero que excede la fecha de máxima floración del cultivo (Figura 2A).

¿Cómo se explica el incremento en los valores de preferencia por la recolección de polen de soja, incluso después del día de la mayor floración del cultivo?

Proponemos dos posibilidades: A) Este proceder puede deberse a un aprendizaje consolidado en la memoria a largo plazo de las abejas con respecto al uso del recurso. Ese comportamiento podría ralentizar la caída en la preferencia de recolección de polen con respecto a lo esperable. Las colmenas funcionan como centros para el procesamiento de la información recopilada por las abejas pecoreadoras. Esta información es comunicada inmediatamente a sus pares a través de movimientos vibratorios (danzas de abejas) que realizan cuando las abejas llegan de sus vuelos de pecoreo (Von Frisch y Chadwick, 1967). También transmiten información a través de los olores con los que se impregnan las pecoreadoras y que se transfieren a sus congéneres dentro de las colmenas, especialmente a través de la trofalaxia. Este tipo de información permanece registrada en las abejas en su memoria a largo plazo (Farina et al., 2005) y condiciona el comportamiento de recolección de las abejas exploradoras durante un período prolongado de tiempo.

Consecuentemente, la comunicación de una disminución en el suministro de recursos de cultivo a sus pares podría presentar un retraso o "delay" con respecto al momento preciso de disminución en la oferta floral. Si este fuera el caso, dada la disminución en el suministro de polen del cultivo, un mayor registro en la preferencia por el polen de soja solo podría ocurrir debido a un mayor número de abejas que recolectan este recurso. Sin embargo, esto no pudo ser validado estadísticamente ya que el número de abejas capturadas en cada fecha no presentó diferencias estadísticamente significativas $(\mathrm{p}=0,057)$.

B) La segunda explicación propuesta considera la gran capacidad de vuelo de las abejas. En promedio se alejan $1,5 \mathrm{~km}$ de la colmena (SteffanDewenter \& Kuhn, 2003) durante el pecoreo, pero se han registrado distancias de vuelo de hasta 6 km (Hagler et al., 2011). En consecuencia, es

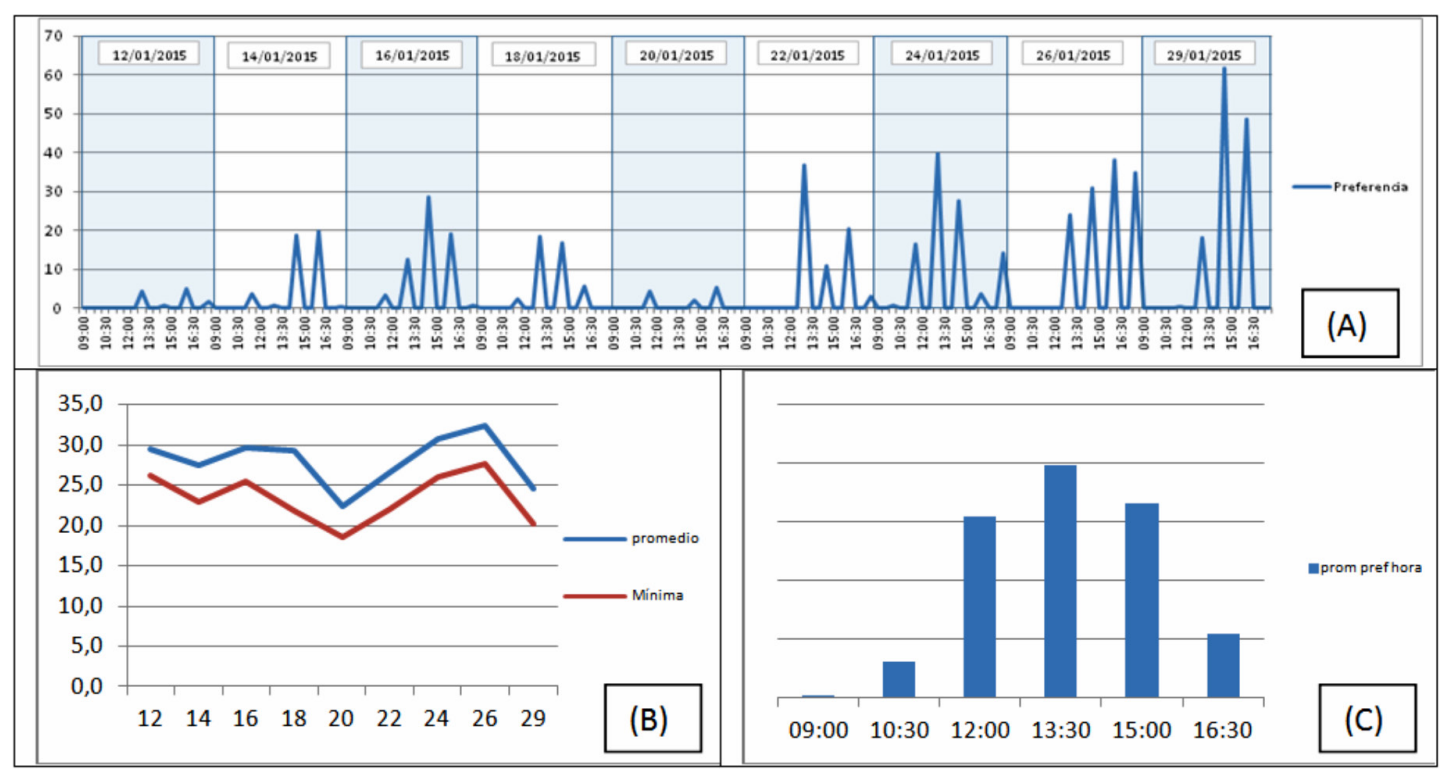

Figura 2. A) Preferencia en cada fecha de muestreo y discriminada por el horario de recolección de polen. B) Registro de la temperatura media y mínima ${ }^{\circ} \mathrm{C}$ durante los días de muestreo y entre 9:00 y 17:00. C) Valores porcentuales promedio del polen de soja corbicular registrado en diferentes horarios de entrada de colmenas durante el período de floración de la soja. 
muy extensa la superficie de exploración de las abejas pecoreadoras $\mathrm{y}$, por consiguiente, no se puede descartar la floración simultánea de soja en lotes agrícolas alejados de las colmenas, que enmascaren una disminución en el suministro de polen de la parcela (experimental) adyacente a las colmenas en las fechas posteriores a la floración máxima de la parcela experimental.

¿Influyen la condición meteorológica y las floraciones alternativas en el comportamiento forrajero de las abejas sobre los cultivos de soja?

Este trabajo muestra una disminución inesperada en la preferencia de recolección de polen de soja el 20 de enero, antes del máximo de floración (Figura 2A).

La explicación de este evento admite dos posibilidades. La primera estaría asociada con el aumento en la oferta de otras flores más atractivas en ese corto período de tiempo. No debe pasarse por alto que la metodología propuesta en este trabajo cuantifica la cantidad de polen de soja que ingresa a las colmenas, pero siempre en relación con el polen total (de todas las especies forrajeadas) de floración concurrente con la de la soja. Y tampoco debemos olvidar que, tal como se resalta en el apartado de Materiales y Métodos, son muchas y variadas las especies vegetales registradas aledañas al cultivo.

La segunda posible explicación a esta inusual disminución de preferencia registrada el día 20 tiene que ver con la meteorología. Precisamente en el día de la mayor disminución en la preferencia por el polen de soja, hay una marcada caída en las temperaturas mínimas y promedio comparativamente con el resto de los días evaluados, lo que presumiblemente sería el factor que altera el comportamiento esperado (Figura 2B). Este proceder podría ser una combinación de dos o más factores, lo que resalta la multiplicidad de elementos influyentes en el comportamiento durante el forrajeo de las abejas y consolida la necesidad de enfoques multidimensionales y con diferentes metodologías de obtención de datos.

La recolección relativa de polen de soja (evaluada a través del \% de polen de soja con respecto al total ingresado en las colmenas) presenta diferencias $(\mathrm{p}<0,001)$ asociadas con el horario de muestreo. Particularmente, la proporción de polen de soja se maximiza y registra diferencias estadísticamente significativas, entre las 12:00 y las 15:00 horas. En este sentido, la Figura 2C muestra los horarios predilectos de las abejas para la recolección de polen de soja, considerando los valores ponderados (\% de polen de soja y número de abejas capturadas).

La preferencia por recolectar el polen de soja en ciertos horarios, sumado a la mayor actividad de alimentación en determinadas horas del día, permite construir el siguiente histograma (Figura 3) que resume de manera elocuente la relativamente baja predilección de Apis mellifera por el polen de soja en relación con el resto de la oferta polínica de la zona. En más del $80 \%$ de los muestreos, el valor de preferencia para el polen de soja es inferior al 20,6\% con respecto al polen total recolectado. En consecuencia, solo en raras circunstancias se observan altas preferencias por el polen de soja, y estos valores se registraron principalmente entre las 12:00 y las 15:00 horas.

En otras palabras, durante la mañana y después de las 3:00 pm, cuando la actividad forrajera de las abejas es menor, de cada 5 abejas que ingresaron a las colmenas con polen corbicular, solo una lo hizo con polen de soja. Por otro lado, entre las 12:00 y las 15:00 horas se observaron valores de preferencia por el polen de soja más elevados y esto ocurrió precisamente durante las horas con el mayor registro de actividad forrajera para las abejas.

Por lo tanto, se establece un rango de horas muy sensible (potencialmente riesgoso) para llevar a cabo pulverizaciones sanitarias en cultivos agrícolas de soja cercanos a las colmenas. Solo en una ocasión (el 26 de enero a las 4:30pm) se superó el valor de preferencia esperable para el horario.

La marcada preferencia evidenciada por determinados horarios de recolección de polen

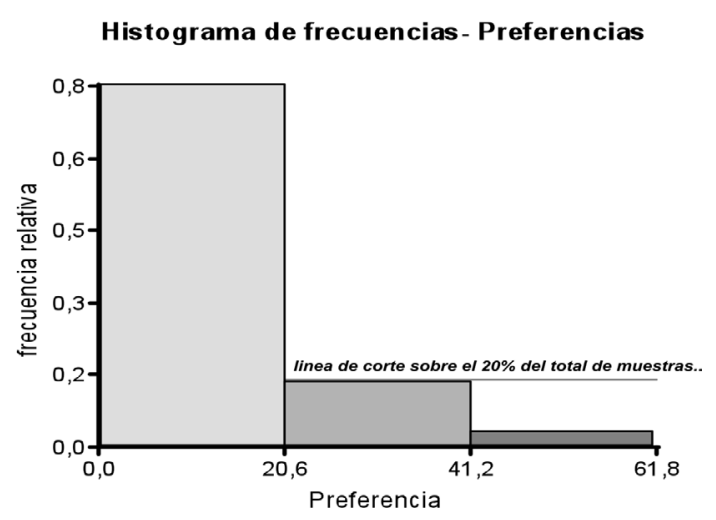

Figura 3. Histograma de preferencias del recurso de polen de soja resultante de las capturas totales de todos los días y horas evaluadas durante el ensayo. 
constituye una herramienta agronómica invaluable, que hace posible reducir las muertes de abejas al mitigar el impacto directo de los pesticidas en sus cuerpos. En regiones muy agriculturizadas, el forrajeo diario de polen constituye una de las principales rutas de contacto de los pesticidas con las colmenas (Rortais et al., 2005) y también posibilita la contaminación de sus productos, ya que el polen retiene la mayor proporción de contaminantes agrícolas que las abejas ingresarán a sus colmenas (Stoner y Eitzer, 2013).

Sin embargo, no debe olvidarse que este es un comportamiento promedio y local. En ciertas circunstancias climáticas o debido a la presencia simultánea de otras floraciones, puede presentar variaciones que evitan arribar a resultados extrapolables a otras regiones con respecto a los horarios en que las abejas recolectan polen del cultivo de soja. Sin embargo, en términos generales, estos resultados constituyen una buena herramienta de mitigación de los efectos adversos para las abejas de las pulverizaciones sanitarias que demandan los cultivos de soja.

\section{Conclusiones}

Los resultados alcanzados aportan una información que debe considerarse para trazar estrategias de gestión agronómica destinadas a reducir el impacto de los agroquímicos en las colmenas adyacentes a los cultivos de soja y/o mejorar las visitas de los polinizadores a los cultivos gestionados por los servicios de polinización. Son conclusiones particularmente relevantes a la luz de recientes investigaciones que muestran aumentos significativos en el rendimiento de grano en cultivos de soja bajo los efectos de la polinización entomófila promovida por Apis mellifera (Blettler et al., 2018).

Estos resultados deberían considerarse durante la redacción de las regulaciones sanitarias de los cultivos y la legislación agrícola, particularmente lo inherente a las pulverizaciones con fitosanitarios.

Las conclusiones y las estrategias agronómicas que se derivan de ellas podrían ser relevantes en otros cultivos extensivos, además de la soja, o incluso deberían considerarse para reducir el impacto negativo de los pesticidas en los polinizadores nativos, además de las abejas melíferas. Obviamente, este punto debería abordarse de manera más profusa en futuras investigaciones, pero el método utilizado se presenta como una posibilidad prometedora, especialmente para el estudio de colonias de abejas no gestionadas donde resultaría imposible recolectar polen por medio de trampas de piquera.

En futuros trabajos que aborden el tema sería importante hacer una lista de las especies cuya oferta y preferencia de polen coincide con la del cultivo objetivo (en fecha y hora). De esta manera se puede llevar a cabo un control adecuado de las especies (tratándose de malezas), para evitar que las abejas desvíen su actividad de pecoreo hacia ellas, reduciendo las visitas al cultivo en el que se presta el servicio de polinización. Por el contrario, la siembra de soja se puede sincronizar para que su floración coincida con la floración de especies de plantas naturalizadas aledañas al cultivo, si lo que se desea es reducir el impacto no deseado de las pulverizaciones en los polinizadores cuando se llevan a cabo en la floración del cultivo.

\section{Literatura citada}

Abrol, D.P.

2007. Honeybees and rapeseed: a pollinator-plant interaction. Advances in botanical research, 45: 337-367.

Basilio, A.

2000. Cosecha polínica por Apis mellifera (Hymenoptera) en el Bajo Delta del Paraná: comportamiento de las abejas y diversidad del polen. Revista del Museo Argentino de Ciencias Naturales nueva serie, 2(2): 111-121.

Blettler, D.C.; Fagúndez, G.A.; Caviglia, O.P.

2018. Contribution of honeybees to soybean yield. Apidologie, 1-11.

Blettler, D.; Fagúndez, G.; Chémez, M.D.

2016. A study of the foraging schedule of honeybees on soy crops as an agronomical tool to mitigate the effects of agrochemicals. Forensic Sci. Int., 7: 14-28.
Di Rienzo, J.A.; Casanoves, F.; Balzarini, M.G.; González, L.; Tablada, M.; Robledo, C.W. InfoStat versión.

2013. Grupo InfoStat, FCA, Universidad Nacional de Córdoba, Argentina. Disponible en: http://www.infostat. com.ar

Dreller, C.; Page, R.E.; Fondrk, M.K.

1999. Regulation of pollen foraging in honeybee colonies: effects of young brood, stored pollen, and empty space. Behav Ecol Sociobiol, 45: 227-233.

Fagúndez, G.A.; Blettler, D.C.; Krumrick, C.G.; Bertos, M.A.; Trujillo, C.G.

2016. Do agrochemicals used during soybean flowering affect the visits of Apis mellifera L.?. Spanish Journal of Agricultural Research, 14(1): 0301.

Farina, W.M.; Grüter, C.; Díaz, P.C. 
2005. Social learning of floral odours inside the honeybee hive. Proceedings of the Royal Society B: Biological Sciences, 272(1575): 1923-1928.

Free, J.B.

1993. Insect pollination of crops. London. Academic Press. $684 \mathrm{p}$.

Gurini, L.; Basilio, A.

1995. Flora apícola en el Delta del Paraná. Darwiniana, 33: 337-346.

Hagler, J.R.; Mueller, S.; Teuber, L.R.; Machtley, S.A.; Van

Deynze, A.

2011. Foraging range of honey bees, Apis mellifera, in alfalfa seed production fields. Journal of Insect Science, 11: 144.

Harano, K.; Sasaki, M.

2015. Adjustment of honey load by honeybee pollen foragers departing from the hive: the effect of pollen load size. Insectes sociaux, 62(4): 497-505.

Hoiss, B.; Krauss, J.; Steffan Dewenter, I.

2015. Interactive effects of elevation, species richness and extreme climatic events on plant-pollinator networks. Global change biology, 21(11): 4086-4097.

Klein, A.M.; Vaissiere, B.E.; Cane, J.H.; Dewenter, I.S.;

Cunningham, S.A.; Kremen, C.; Tscharntke, T.

2007. Importance of pollinators in changing landscapes for world crops. Proceedings of the Royal Society B: Biological Sciences, 274: 303-313.

Mullin, C.A.; Frazier, M.; Frazier, J.L.; Ashcraft,S., Simonds,

R.; vanEngelsdorp,D.; Pettis, J.S.

2010. High levels of miticides and agrochemicals in North American apiaries: implications for honey bee health, PloS ONE, 5(3) Article ID e9754.

Rader, R.; Edwards, W.; Westcott, D.A.; Cunningham, S.A.; Howlett, B.G.

2013. Diurnal effectiveness of pollination by bees and flies in agricultural Brassica rapa: Implications forecosystem resilience. Basic and Applied Ecology, 14: 20-27.
Rodríguez-Parilli, S.A.

2014. Abejas (Hymenoptera: Apoidea) visitantes de Waltheria americana L. (Sterculaceae), enárea agrícola de la Universidad Rómulo Gallegos, Guárico, Venezuela. Zootecnia Tropical, 29(3).

Rortais, A.; Arnold, G.; Halm, M.P.; Touffet-Briens, F. 2005. Modes of honeybees exposure to systemic insecticides: estimated amounts of contaminated pollen and nectar consumed by different categories of bees. Apidologie, 36(1), 71-83.

Shawer, M.B.

1987. Mayor pollen sources in Kafr El-Seikh Egypt and the effect of pollen supply on broad area and honey yield. $J$. of Apic. Res., 26 (1): 43-46.

Steffan-Dewenter, I.; Kuhn, A.

2003. Honeybee foraging in differentially structured landscapes. Proceedings of the Royal Society of London. Series B: Biological Sciences, 270(1515): 569-575.

Stoner, K.A.; Eitzer, B.D.

2013. Using a hazard quotient to evaluate pesticide residues detected in pollen trapped from honey bees (Apis mellifera) in Connecticut. PLoS One, 8(10): e77550.

Torretta, J.P.; Medan, D.; Roig Alsina, A.; Montaldo, H.N. 2010. Visitantes florales diurnos del girasol (Helianthus annuus, Asterales: Asteraceae) en la Argentina. Revista de la Sociedad Entomológica Argentina, 69(1-2):17-32.

Vaissière, B.E.; Vinson, S.B.

1994. Pollen morphology and its effect on pollen collection by honey bees, Apis Mellifera L. (Hymenoptera: Apidae), with special Reference to Upland Cotton, Gossypium Hirsutum L. (Malvaceae). Grana, 33(3): 128-138.

Viglizzo, E.F.; Frank, F.C.; Carreño, L.V.; Jobbágy, E.G.; Pereyra, H.; Clatt, J.; Pincén, D.; Ricard, M.F.

2010. Ecological and environmental footprint of 50 years of agricultural expansion in Argentina. Global Change Biology, 17: 959-973.

Von Frisch, K.; Chadwick, L.E. 1967.

The dance language and orientation of bees (Vol. 1). Belknap Press of Harvard University Press. Cambridge, MA. US. 
